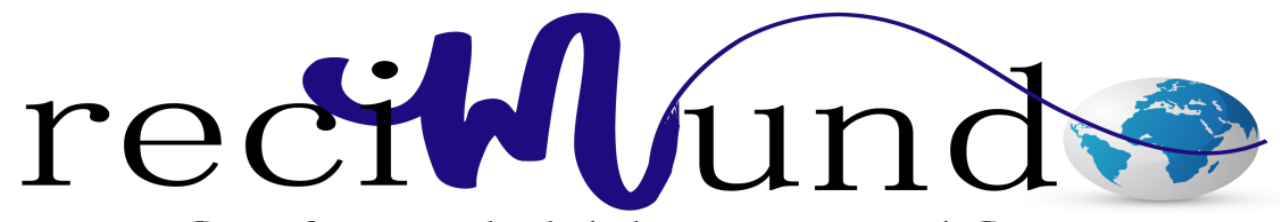

Revista Científica Mundo de la Investigación y el Conocimiento

Héctor Danilo Hugo Ullauri ${ }^{\text {a }}$ Juan Carlos Torres Espinoza ${ }^{\text {b }}$; Víctor Eulogio Parraga Trejo ${ }^{\mathrm{c}}$

La ordenación del territorio en Ecuador. Evaluación del plan de desarrollo y ordenamiento territorial parroquial de virgen de Fátima

Revista Científica Mundo de la Investigación y el Conocimiento. Vol. 1 núm., 5, diciembre, 2017, pp. 138-146

DOI: $10.26820 /$ recimundo/1.5.2017.138-146

Editorial Saberes del Conocimiento
a. Universidad de Guayaquil; hector.hugou@ug.edu.ec
b. Universidad de Guayaquil; juan.torreses@ug.edu.ec
c. Universidad de Guayaquil; victor.parragat@ug.edu.ec 


\section{La ordenación del territorio en Ecuador. Evaluación del plan de desarrollo y ordenamiento territorial parroquial de virgen de Fátima}

Vol. 1, núm. 5., (2017)

Héctor Danilo Hugo Ullauri; Juan Carlos Torres Espinoza; Víctor Eulogio Parraga Trejo

\section{RESUMEN}

El objetivo principal de la investigación fue evaluar la ley de ordenamiento territorial, uso y gestión de suelo de Ecuador, a través del caso de estudio plan de desarrollo y ordenamiento territorial del GAD parroquial de Virgen de Fátima, para ello se caracterizó la planificación territorial en Ecuador, prestando atención tanto a la legislación de partida como a los instrumentos de planificación que se derivan de la misma, luego se diagnosticó el plan de desarrollo y ordenamiento territorial parroquial vigente de Virgen de Fátima (PDOTVF), y la regulación normativa e instrumental que estructura la Ley orgánica de ordenamiento territorial, uso y gestión de suelo (LOOTUGS). Finalmente se emitieron líneas de mejora desde un análisis territorial del caso de estudio, para potenciar la viabilidad que permita ejecutar efectivamente los instrumentos de ordenamiento territorial. La metodología aplicada, es estudio de caso, en la cual planteamos el problema y objetivos, posteriormente la revisión de literatura existente sobre el tema, se triangula las fuentes de información de medios digitales, entrevistas, y documentos oficiales, posterior a ello se realizó la transcripción de los datos, y un análisis global y profundo, la valoración global y la propuesta de mejora. Los resultados del análisis al PDOT, arroja deficiencias en cuanto a la articulación de los planes, programas y proyectos con el medio físico los cuales desconocen el sistema territorial y se limita a promulgar estrategias territoriales que dependen de otras instancias de gobierno.

Palabras clave: Ordenamiento; territorial; desarrollo; suelo; planificación. 


\title{
La ordenación del territorio en Ecuador. Evaluación del plan de desarrollo y ordenamiento territorial parroquial de virgen de Fátima
}

Vol. 1, núm. 5., (2017)

Héctor Danilo Hugo Ullauri; Juan Carlos Torres Espinoza; Víctor Eulogio Parraga Trejo

\begin{abstract}
The main objective of the investigation was to evaluate the law of territorial ordering, use and land management of Ecuador, through the case study plan development and territorial ordering of the parochial GAD of Virgen de Fátima, for it was characterized the territorial planning in Ecuador, paying attention to both the legislation of departure and the planning instruments derived from it, then diagnosed the development plan and current parochial territorial ordinance of Virgen de Fátima (PDOTVF), and the normative and instrumental regulation that structure the Organic Law of land use, land use and management (LOOTUGS). Finally lines of improvement were issued from a territorial analysis of the case study, to enhance the feasibility that allows to effectively implement the instruments of territorial ordering. The applied methodology is a case study, in which we pose the problem and objectives, then review the existing literature on the subject, triangulate the sources of information from digital media, interviews, and official documents, after which the transcription of the data, and a global and deep analysis, the global assessment and the improvement proposal. The results of the analysis to the PDOT, shows deficiencies in the articulation of the plans, programs and projects with the physical environment, which ignore the territorial system and limits itself to enacting territorial strategies that depend on other instances of government.
\end{abstract}

Keywords: Land management; territorial; development; land; planning. 


\section{La ordenación del territorio en Ecuador. Evaluación del plan de desarrollo y ordenamiento territorial parroquial de virgen de Fátima}

Vol. 1, núm. 5., (2017)

Héctor Danilo Hugo Ullauri; Juan Carlos Torres Espinoza; Víctor Eulogio Parraga Trejo

\section{Introducción.}

Ecuador carece de una ley específica sobre ordenación territorial, carencia que no fue compensada por los instrumentos legales que la sustituyeron. Esto conllevo, a que se ordene el uso de suelo en el medio rural en el plan provincial, pero también en los planes cantonales e incluso en los de las parroquiales rurales.

El presente trabajo nace por la necesidad de contribuir en el desarrollo de los asentamientos humanos ubicados en la parroquia Virgen de Fátima. Los instrumentos de ordenamiento territorial vigentes no responden a las especificidades del sistema territorial. El objeto de estudio es el ordenamiento territorial y el campo de investigación es el Plan de desarrollo y ordenamiento territorial (PDOT) parroquial de Virgen de Fátima. (Gobierno Autónomo Descentralizado Parroquial de Virgen de Fátima, 2015)

En los textos constitucionales es muy clara la idea de simultaneidad en cuanto a la redacción de estos instrumentos, lo que es absolutamente pertinente desde el punto de vista metodológico además de la optimización de recursos y una razonable simplificación de los procedimientos de participación ciudadana, aprobación y difusión; lo mismo puede decirse del plan nacional de desarrollo y de la estrategia territorial nacional prevista por el Código orgánico de planificación y finanzas públicas (COPFP)

En Ecuador, los lineamientos metodológicos para la elaboración de Planes de Desarrollo y Ordenamiento Territorial (PDOT) han sido impuestos a partir de estándares nacionales, y se confrontan con las restricciones técnicas y operativas para su ejecución por parte de los gobiernos autónomos descentralizados. (Asamblea Nacional de Ecuador, 2016) 


\section{La ordenación del territorio en Ecuador. Evaluación del plan de desarrollo y ordenamiento territorial parroquial de virgen de Fátima}

Vol. 1, núm. 5., (2017)

Héctor Danilo Hugo Ullauri; Juan Carlos Torres Espinoza; Víctor Eulogio Parraga Trejo

\section{Materiales y Métodos}

Para el desarrollo del presente trabajo, la sistematización de la información estará dividida de la siguiente manera:

La primera, recoge el marco teórico y conceptual básico relacionado con el ordenamiento territorial.

En la segunda parte, se realizará un análisis sistemático y comparativo de la información recopilada sobe el marco normativo e instrumental de la ordenación del territorio en Ecuador, realizando una comparación con la Parroquia Virgen de Fátima.

A continuación de lo anterior, se realizará una tercera parte de Análisis crítico de la Ley Orgánica de Ordenamiento Territorial, uso y gestión de suelo de Ecuador, aprobada el 12 de mayo de 2016 y el Plan de Desarrollo y Ordenamiento Territorial parroquial de Virgen de Fátima (PDOTVF).

Este proceso implica una investigación de carácter cualitativo, el tamaño de la muestra no es importante desde una perspectiva probabilística en la que se da prioridad a la calidad frente a la cantidad de los datos. (Martínez Carazo, 2006) 


\section{La ordenación del territorio en Ecuador. Evaluación del plan de desarrollo y ordenamiento territorial parroquial de virgen de Fátima}

Vol. 1, núm. 5., (2017)

Héctor Danilo Hugo Ullauri; Juan Carlos Torres Espinoza; Víctor Eulogio Parraga Trejo

\section{Resultados.}

Tabla $N^{\circ}$ 1: Caracterización de la planificación territorial en el Ecuador

\begin{tabular}{|c|c|}
\hline NIVELES & INSTRUMENTOS \\
\hline SUPRANACIONAL & Plan Fronterizo/Binacional/Regional \\
\hline NACIONAL & $\begin{array}{l}\text { Estrategia territorial nacional/Plan especial para proyecto nacional } \\
\text { de carácter estratégico/plan sectorial ejecutivo }\end{array}$ \\
\hline \multirow{4}{*}{$\begin{array}{l}\text { REGIONAL, } \\
\text { PROVINCIAL, } \\
\text { CANTONAL, } \\
\text { PARROQUIA } \\
\text { RURAL }\end{array}$} & $\begin{array}{c}\text { Plan de desarrollo y ordenamiento territorial/pla } \\
\text { complementario/Plan para el desarrollo sustentable y ordenamiento } \\
\text { territorial }\end{array}$ \\
\hline & Plan de uso y gestión del suelo (Vigencia de 12 años) \\
\hline & $\begin{array}{l}\text { Plan urbanístico complementario(Plan maestro sectorial, Plan } \\
\text { parcial) }\end{array}$ \\
\hline & $\begin{array}{l}\text { Otros instrumentos de planeamiento urbanístico (en función a } \\
\text { características del territorio) }\end{array}$ \\
\hline
\end{tabular}

Fuente: Elaboración Propia. (Gómez Orea \& Gómez Vallarino, 2013)

Los nuevos instrumentos de planificación, son una oportunidad para mejorar, se debe entender el territorio como un organismo vivo, que necesita de efluentes, y emite afluentes que finalmente llegan a la estructura de soporte, el medio físico, y depende de nuestro accionar que las emisiones vertidas al agua, aire y tierra, no superen su capacidad de autodepuración. (del Territorio, 1983)

Sobre los problemas y potencialidades constan en el ámbito de estudio del presente proyecto, fueron identificados mediante el análisis por componentes biofísico, económico, social-cultural, político institucional, asentamientos humanos y movilidad, conectividad y energía. Se determina las inequidades y desequilibrios territoriales presentes en el caso de estudio. (Orea, 1985) 


\section{La ordenación del territorio en Ecuador. Evaluación del plan de desarrollo y ordenamiento territorial parroquial de virgen de Fátima}

Vol. 1, núm. 5., (2017)

Héctor Danilo Hugo Ullauri; Juan Carlos Torres Espinoza; Víctor Eulogio Parraga Trejo

Dentro de los mecanismos que regula la Ley Orgánica De Ordenamiento Territorial, Uso y

Gestión del Suelo. (LOOTUGS) (Asamblea Nacional de Ecuador, 2016) para que sean implementados por los Gobiernos Autónomos Descentralizados (GAD) se encuentra la clasificación y subclasificación de los suelos urbanos y rurales y el planeamiento que sobre los mismos deben ejecutar los gobiernos locales. Esta clasificación tiene como base las características de los espacios territoriales, en relación a los servicios, equipamientos e infraestructuras que poseen, para promover el desarrollo urbanístico. (Elorrieta, Jorge , Cantos , \& Sánchez, 2016)

En relación a los instrumentos para la gestión del suelo destacan la distribución equitativa de cargas y beneficios, que obliga a los propietarios del suelo, según sus características, a realizar diversas acciones para el aprovechamiento urbanístico (cesión gratuita de suelo destinado a espacio público) y el financiamiento de los gastos derivados de las reubicaciones y costos de planificación, a cambio de beneficios derivados del aprovechamiento urbanístico y compensaciones por las cargas impuestas. (Willems \& Diaz Navarrete, 1997)

Sobre la propuesta de mejora para la eficiente implementación de los mecanismos, la LOOTUGS (Asamblea Nacional de Ecuador, 2016) regula la creación de dos entidades: Consejo Técnico y Superintendencia. El primero para emitir regulaciones técnicas complementarias, y el segundo para vigilar el cumplimiento de las disposiciones por parte de los GAD. Adicionalmente, se fortalece el accionar de los $\mathrm{GAD}$, con la especificación de infracciones que pudieran cometer las personas naturales y jurídicas, públicas y privadas, en relación al uso y gestión del suelo, y las sanciones respectivas. (Orea, 1985) 


\section{La ordenación del territorio en Ecuador. Evaluación del plan de desarrollo y ordenamiento territorial parroquial de virgen de Fátima}

Vol. 1, núm. 5., (2017)

Héctor Danilo Hugo Ullauri; Juan Carlos Torres Espinoza; Víctor Eulogio Parraga Trejo

\section{Conclusiones}

- Del presente estudio, se concluye que los instrumentos de ordenamiento territorial usados en la actualidad, no responden a las condicionantes, socioeconómicas y del medio físico, son muy generales y complejas para el desarrollo de proyectos específicos.

- Para el caso puntual de Virgen de Fátima, se recomienda la elaboración de un Plan de uso y gestión de suelo, donde a partir del análisis de los componentes estructurante y urbanístico, puedan definirse los polígonos de intervención urbana y rural. Y en ese caso ordenar el territorio a partir de dichos polígonos mediante plan parcial.

\section{Bibliografía}

Asamblea Nacional de Ecuador. (2016). Proyecto de Ley orgánica de Ordenamiento Territorial, Uso Y gestión de suelo. Ecuador.

del Territorio, C. E. (1983). onferencia Europea de Ministros Responsables de Política Regional y Ordenación del territorio. Torremolinos.

Elorrieta, B., Jorge , S., Cantos , O., \& Sánchez, D. (2016). La sostenibilidad en la planificación territorial de escala regional en España: estudio de casos. Cuadernos Geográficos(55), $149-175$.

Gobierno Autónomo Descentralizado Parroquial de Virgen de Fátima. (2015). Actualización del Plan de Desarrollo y Ordenamiento Territorial. Virgen de Fátima. 


\section{La ordenación del territorio en Ecuador. Evaluación del plan de desarrollo y ordenamiento territorial parroquial de virgen de Fátima}

Vol. 1, núm. 5., (2017)

Héctor Danilo Hugo Ullauri; Juan Carlos Torres Espinoza; Víctor Eulogio Parraga Trejo

Gómez Orea, D., \& Gómez Vallarino, A. (2013). Ordenación Territorial. Madrid: Ediciones Mundi-Prensa, Ed.

Martínez Carazo, P. C. (2006). El método de estudio de caso: Estrategia metodológica de la investigación científica. Pensamiento Y Gestión: Revista de La División de Ciencias Administrativas de La Universidad Del Norte, 20, 165-193.

Orea, D. G. (1985). El espacio rural en la ordenación del territorio. (P. y. Instituto de Estudios Agrarios, Ed.) Recuperado el 10 de 2017, de Repositorio Digital del Instituto Andaluz del Patrimonio Historico: http://hdl.handle.net/11532/222315

Willems, P., \& Diaz Navarrete, G. (1997). Herramientas de SIG como soporte a la Planificación Territorial dentro del estudio de Ordenamiento Territorial de las laderas sur-orientales del volcán Pichincha en Quito, Ecuador. X Congreso de Métodos Cuantitativos, Sistemas de Información Geográfica y Teledetección. Ciencia y Tecnología de la Información Geográfica en un Mundo Globalizado. Universidad de Valladolid, Asociación de Geógrafos Españoles, (págs. 1-17). 